\title{
Problems of Homeomorphism Arising in the Theory of Grid Generation
}

\author{
M. F. Prokhorova ${ }^{1}$
}

Received November 1, 2007

\begin{abstract}
Some general criteria of being a homeomorphism for continuous maps of topological spaces and topological manifolds are proved in this paper, as well as criteria of being a diffeomorphism for smooth maps of smooth manifolds.
\end{abstract}

DOI: $10.1134 /$ S0081543808050155

\section{INTRODUCTION}

While developing algorithms of grid generation for numerical calculations in domains of complicated configuration, it is necessary to apply (see, for example, $[1,2]$ ) different criteria for finding out whether a continuous map is a homeomorphism or whether a smooth map is a diffeomorphism. Such criteria for bounded domains in $\mathbb{R}^{n}$ were suggested in $[3,4]$. Unfortunately, some theorems in $[3,4]$ are incorrect, while the proofs of some others are incomplete (see Section 6).

In this paper we formulate and prove some general criteria of being a homeomorphism for continuous maps of topological spaces, topological manifolds, and triangulated topological manifolds, as well as criteria of being a diffeomorphism for smooth maps of smooth manifolds. This paper continues [5].

For the convenience of the reader who deals not with topology but with numerical methods, in the paper we give definitions of the main topological notions mentioned in theorem formulations. It is possible to consider them in more detail, for example, in [6,7]. Also for the reader's convenience, at the beginning of Section 1 there are proofs of some elementary topological statements widely used in the subsequent sections. In Section 1 we describe some criteria of whether a continuous map from one topological space to another is an immersion, embedding, or homeomorphism. In Section 2 we consider continuous maps of topological manifolds. In Section 3 we consider continuous maps of topological manifolds with a singularity on an embedded cell space, as well as continuous maps of topological manifolds equipped with a cell decomposition. In Section 4 we consider continuous maps of triangulated topological manifolds and pseudomanifolds. In Section 5 we mention some evident corollaries from the results of the preceding sections for the case of smooth and piecewisesmooth maps. In Section 6 we give a short comparison of the results of this paper with the results of $[3,4]$.

\footnotetext{
${ }^{1}$ Institute of Mathematics and Mechanics, Ural Branch of the Russian Academy of Sciences, ul. S. Kovalevskoi 16, Yekaterinburg, 620219 Russia

e-mail: pmf@imm.uran.ru
} 


\section{TOPOLOGICAL SPACES}

\section{Notation.}

- Let $A, A_{1}, \ldots, A_{k}$ be subspaces of $X$, and let $B, B_{1}, \ldots, B_{k}$ be subspaces of $Y$ (a subspace is a subset equipped with topology induced from the basic space). Let us define the following: $C(X, Y)$ is the set of continuous maps from $X$ to $Y$, $C(X, A ; Y, B)$ is the set of maps $f \in C(X, Y)$ such that $f(A) \subset B$, $C\left(X, A_{1}, \ldots, A_{k} ; Y, B_{1}, \ldots, B_{k}\right)$ is the set of $f \in C(X, Y)$ such that $f\left(A_{i}\right) \subset B_{i}$ for all $i=1 \ldots k$.

- For $f \in C(X, A ; Y, B)$ let us denote by $\left.f\right|_{A \rightarrow B}$ the map obtained from $f$ by restricting the domain to $A$ and the range to $B$.

Let us note that maps $f=\left.f\right|_{X \rightarrow Y},\left.f\right|_{A}=\left.f\right|_{A \rightarrow Y}$, and $\left.f\right|_{A \rightarrow B}$ are different and may possess different properties. For example, the map $f: \mathbb{R} \rightarrow \mathbb{R}$ given by the formula $f(x)=\sin x$ is not open, and $\left.f\right|_{\mathbb{R} \rightarrow[-1,1]}$ is open.

Definition 1. Let $X$ and $Y$ be topological spaces. We will call a map $f: X \rightarrow Y$

- an embedding if $\left.f\right|_{X \rightarrow f(X)}$ is a homeomorphism,

- an immersion if any point $x \in X$ has a neighborhood $U$ such that $\left.f\right|_{U \rightarrow f(U)}$ is a homeomorphism,

- a local homeomorphism if any point $x \in X$ has a neighborhood $U$ such that $f(U)$ is open in $Y$ and $\left.f\right|_{U \rightarrow f(U)}$ is a homeomorphism.

\section{Remarks.}

1. All embeddings, immersions, and local homeomorphisms are continuous.

2. Embeddings and local homeomorphisms are immersions.

3. A map is a local homeomorphism if and only if it is an open immersion.

Everywhere further $X$ and $Y$ are Hausdorff topological spaces satisfying the second axiom of countability.

Let us recall that a continuous map $f: X \rightarrow Y$ is called proper if for any compact set $K \subset Y$ its preimage $f^{-1}(K)$ is compact. For a compact set $X$, any continuous map $f: X \rightarrow Y$ is proper.

A continuous map $f: X \rightarrow Y$ is called a covering if any point $y \in Y$ has a neighborhood $V$ such that $f$ maps every connected component of $f^{-1}(V)$ to $V$ homeomorphically.

Lemma 1. Let $Y$ be connected, and let $f: X \rightarrow Y$ be a proper local homeomorphism. Then $f$ is a covering.

Proof. For any $y \in \operatorname{Im} f$ there exists a neighborhood $V \ni y$ such that $V \subset \operatorname{Im} f$, because $f$ is a local homeomorphism. Thus, $\operatorname{Im} f$ is open in $Y$. If $\operatorname{Im} f \neq Y$, let us take an arbitrary point $y$ on the boundary of $\operatorname{Im} f$. Let us choose $y_{n} \in \operatorname{Im} f, y_{n} \rightarrow y, x_{n} \in f^{-1}\left(y_{n}\right)$. The set $\bigcup\left\{y_{n}\right\} \cup\{y\}$ is compact, thus its preimage $K$ is also compact. From the sequence $\left\{x_{n}\right\} \subset K$, we can choose a subsequence $\left\{x_{n}^{\prime}\right\} \rightarrow x \in K$. However, $f(x)=\lim f\left(x_{n}^{\prime}\right)=y$; hence, $y \in \operatorname{Im} f$. Thus, $f$ is surjective.

Let us take an arbitrary point $y \in Y$. The preimage of $y$ is discrete (because $f$ is a local homeomorphism) and compact; hence, it is finite. 
Let $f^{-1}(y)=\left\{x_{i}: i \in I\right\}$ ( $I$ is finite), and let $U_{i}$ be disjoint neighborhoods of the points $x_{i}$, which are projected homeomorphically to open sets in $Y ; V=\bigcap f\left(U_{i}\right)$.

Let us show that there exists a neighborhood $W \ni y$ such that its preimage is contained in $\bigcup U_{i}$. If it is not so, then there exist $z_{k} \notin \bigcup U_{i}, k \in \mathbb{N}$, such that $f\left(z_{k}\right) \rightarrow y$. Since the set $\bigcup\left\{f\left(z_{k}\right)\right\} \cup\{y\}$ is compact, its preimage $K$ is also compact. From the sequence $\left\{z_{k}\right\} \subset K$, we can choose a subsequence $\left\{z_{k}^{\prime}\right\} \rightarrow z \in K$. However, $f(z)=\lim f\left(z_{k}^{\prime}\right)=y$; hence, $z=x_{i}$ for some $i$, and $z_{k}^{\prime}$ are in $U_{i}$ starting from some $k=k_{0}$, which contradicts the hypothesis.

Thus, there exists a neighborhood $W \ni y$ such that its preimage is a disjoint union of domains $W_{i}=f^{-1}(W) \cap U_{i}$, and each of them is projected to $W$ homeomorphically. Thus, $f$ is a covering.

The next two lemmas follow immediately from Lemma 1 and from elementary properties of coverings; for the sake of the reader's convenience we give their complete proofs here.

Lemma 2. Suppose that $f: X \rightarrow Y$ is a proper local homeomorphism, $Y$ is connected, and there exists a point $y_{0} \in Y$ with a one-point preimage: $f^{-1}\left(y_{0}\right)=\left\{x_{0}\right\}$. Then $f$ is a homeomorphism.

Proof. Let us consider the map $\varphi: Y \rightarrow \mathbb{Z}_{+}, \varphi(y)=\operatorname{card} f^{-1}(y)$. As $f$ is a covering, $\varphi$ is locally constant. However, $Y$ is connected, which means that $\varphi$ is globally constant and identically equal to $\varphi\left(y_{0}\right)=1$. Thus, the map $f: X \rightarrow Y$ is bijective. Since it is a local homeomorphism, we conclude that it is a homeomorphism.

Lemma 3. Let $X$ be path-connected, let $Y$ be connected and simply connected, and let $f: X \rightarrow$ $Y$ be a proper local homeomorphism. Then $f$ is a homeomorphism.

Proof. Let us assume that there exist two different points $x_{0}, x_{1} \in X$ with coinciding images: $f\left(x_{0}\right)=f\left(x_{1}\right)=y$. Since $X$ is path-connected, there exists a continuous curve $\gamma:[0,1] \rightarrow X$, $\gamma(0)=x_{0}, \gamma(1)=x_{1}$. As $Y$ is simply connected, there exists a homotopy $h_{t}:[0,1] \rightarrow Y, h_{0}=f \circ \gamma$, $h_{t}(0)=h_{t}(1)=h_{1}(s) \equiv y$. According to Lemma 1, $f$ is a covering. Hence, the homotopy $h_{t}$ lifts to the homotopy $H_{t}:[0,1] \rightarrow X$, so that $f \circ H_{t}=h_{t}, H_{0}=\gamma[7]$. Let us consider the curve $H_{1}(s)$ connecting the points $x_{0}$ and $x_{1}$. Its projection to $Y$ is the point $y$. However, this is contrary to the condition that $f$ is locally homeomorphic at $x_{0}$ (some neighborhood of the point $x_{0}$ does not contain points that are projected to $y$ and differ from $x_{0}$ ). Thus, $f$ is an injection. As $Y$ is connected and $f$ is a covering, $f$ is surjective and hence it is a homeomorphism.

Lemma 4. Let $f: X \rightarrow Y$ be a continuous proper injective map. Then $f$ is an embedding.

Proof. As $X$ satisfies the second axiom of countability, it is sufficient to prove that if $y_{n}, y \in$ $\operatorname{Im} f$ and $\left\{y_{n}\right\} \rightarrow y$, then the sequence $x_{n}=f^{-1}\left(y_{n}\right)$ converges to $x=f^{-1}(y)$. Since the set $K=\bigcup\left\{y_{n}\right\} \cup\{y\}$ is compact, $f^{-1}(K)$ is also compact, and the sequence $\left\{x_{n}\right\}$ has a limit point in $f^{-1}(K)$. If $x^{\prime}$ is a limit point of $\left\{x_{n}\right\}$ in $X$, then $f\left(x^{\prime}\right)$ is a limit point of $\left\{y_{n}\right\}$, and $x^{\prime}=x$. Thus, $\lim x_{n}=x$; hence, $f^{-1}: \operatorname{Im} f \rightarrow X$ is continuous.

Let us recall that a Hausdorff topological space $X$ is called regular if for any point $x \in X$ and its neighborhood $U$ there exists a neighborhood $V \ni x$ such that its closure is in $U$.

Lemma 5. Let $X$ be a regular topological space, let $X^{\prime} \subset X$ be open, let $f: X \rightarrow Y$ be a continuous proper map, and let $f^{\prime}=\left.f\right|_{X^{\prime}}$ be a local injection (i.e., any point of $X^{\prime}$ has a neighborhood $U$ in $X^{\prime}$ such that the restriction of $f^{\prime}$ to $U$ is an injection). Then $f^{\prime}$ is an immersion.

Proof. Suppose that $x \in X^{\prime}, U \subset X^{\prime}$ is a neighborhood of $x$, and $\left.f^{\prime}\right|_{U}$ is an injection. Let $W$ be a neighborhood of the point $x$ such that its closure $\bar{W}$ is contained in $U$. For any compact set $K \subset f(W)$, the preimage $\left(\left.f\right|_{W}\right)^{-1}(K)=f^{-1}(K) \cap W=f^{-1}(K) \cap \bar{W}$ is compact; hence, $\left.f\right|_{W \rightarrow f(W)}$ is a proper map. According to Lemma 4 , it is an embedding. Consequently, $f^{\prime}$ is an immersion. 
Theorem 1. Suppose that $X$ is regular, $f: X \rightarrow Y$ is a continuous proper map, $A \subset X$, $f(A) \cap f(X \backslash A)=\varnothing,\left.f\right|_{A}$ is an injection, $\left.f\right|_{X \backslash A \rightarrow Y \backslash f(A)}$ is a local homeomorphism, and one of the following conditions is satisfied:

- $Y \backslash f(A)$ is connected, and there exists a point in $Y \backslash f(A)$ with singleton preimage, or

- $X \backslash A$ is path-connected, and $Y \backslash f(A)$ is connected and simply connected.

Then $f$ is a homeomorphism.

Proof. The restriction $\left.f\right|_{X \backslash A \rightarrow Y \backslash f(A)}$ is a proper local homeomorphism, and, according to Lemmas 2 and 3, it is a homeomorphism. Then $f: X \rightarrow Y$ is a continuous bijection, and by Lemma 4 it is a homeomorphism.

Theorem 2. Suppose that $f: X \rightarrow Y$ is a continuous proper map, $A \subset X$, and the restriction of $f$ to $X \backslash A$ is a local homeomorphism.

1. If $Y_{1}=Y \backslash f(A)$ is connected and satisfies one of the conditions

- for some point $y_{0} \in Y_{1}$, its preimage $f^{-1}\left(y_{0}\right)$ is a singleton, or

- $X_{1}=f^{-1}\left(Y_{1}\right)$ is path-connected and $Y_{1}$ is simply connected,

then $\left.f\right|_{X_{1}}$ is an embedding.

2. If $\left.f\right|_{X \backslash f^{-1}(f(A))}$ is an injection and the interior of $f(A)$ in $Y$ is empty, then $\left.f\right|_{X \backslash A}$ is an open embedding.

3. If $\left.f\right|_{X \backslash A}$ is an open embedding, $A$ is closed, its interior in $X$ is empty, and $X / A$ is a Hausdorff space, then $f(A) \cap f(X \backslash A)=\varnothing$. If, in addition, $Y \backslash f(A)$ is connected, then $\left.f\right|_{X \backslash A \rightarrow Y \backslash f(A)}$ is a homeomorphism.

Here $X / A$ is a quotient space of $X$ with the subspace $A$ contracted to a point. Note that the requirement in assertion 2 of the theorem that $X / A$ is a Hausdorff space is satisfied, in particular, if $X$ is regular or if $A$ is compact.

Proof.

1. For any compact set $K \subset Y_{1}$, its preimage $\left(\left.f\right|_{X_{1} \rightarrow Y_{1}}\right)^{-1}(K)=f^{-1}(K)$ is compact because $f$ is proper; hence, $\left.f\right|_{X_{1} \rightarrow Y_{1}}$ is a proper map. Applying Lemmas 2 and 3, we find that $\left.f\right|_{X_{1} \rightarrow Y_{1}}$ is a homeomorphism.

2. Let us consider the set $\left\{y \in Y\right.$ : card $\left.\left(f^{-1}(y) \cap(X \backslash A)\right)>1\right\}$. It is open (because $\left.f\right|_{X \backslash A}$ is a local homeomorphism) and is contained in $f(A)$; hence, it is empty. Thus, $\left.f\right|_{X \backslash A}$ is an open embedding.

3. Suppose that $U \subset X \backslash A$ is an open set and its closure also lies in $X \backslash A$. The set $U^{\prime}=$ $(X \backslash \bar{U}) \cap f^{-1}(f(U))$ is open in $X$ and is contained in $A$. As the interior of $A$ in $X$ is empty, $U^{\prime}$ is also empty, and $f^{-1}(f(U)) \subset \bar{U} \subset X \backslash A$. For any point $x \in X \backslash A, x$ and $A$ have disjoint neighborhoods; hence, the union of all such $U$ coincides with $X \backslash A$. Thus, $f(A) \cap f(X \backslash A)=\varnothing$. The embedding $\left.f\right|_{X \backslash A \rightarrow Y \backslash f(A)}$ is proper and open, and by Lemma 1 it is a homeomorphism on the clopen subset $Y \backslash f(A)$.

Theorem 3. Suppose that $f: X \rightarrow Y$ is a continuous proper map, $X$ is regular, $A \subset X$ is closed, $\operatorname{int}_{X} A=\operatorname{int}_{Y} f(A)=\varnothing,\left.f\right|_{X \backslash A}$ is a local homeomorphism, and $\left.f\right|_{X \backslash f^{-1}(f(A))}$ is an injection. Then

1. If $\left.f\right|_{A}$ is an injection, then $f$ is an embedding; if, in addition, $Y \backslash f(A)$ is connected, then $f$ is a homeomorphism. 
2. If $\left.f\right|_{A}$ is an immersion, then $f$ is also an immersion.

3. If $A^{\prime} \subset A$ is closed and $\left.f\right|_{A \backslash A^{\prime}}$ is an immersion, then $\left.f\right|_{X \backslash A^{\prime}}$ is also an immersion.

Proof. The restriction $\left.f\right|_{X \backslash f^{-1}(f(A)) \rightarrow Y \backslash f(A)}$ is a proper map, and, by Lemma 4, it is an embedding. According to Theorem 2, $\left.f\right|_{X \backslash A}$ is also an embedding, $f(A) \cap f(X \backslash A)=\varnothing$, and $\left.f\right|_{X \backslash A}$ is a homeomorphism onto the clopen subset $Y \backslash f(A)$.

1. The map $f$ is an injection, and, by Lemma 4, it is an embedding. If $Y \backslash f(A)$ is connected, then, by virtue of assertion 3 of Theorem $2, f$ is a bijection, and, by virtue of Lemma 4 , it is a homeomorphism.

2. This assertion is a corollary of the more general assertion 3 of this theorem, which is proved below.

3. Let $x \in A \backslash A^{\prime}$ be an arbitrary point. Let us take a neighborhood $U \ni x$ in $X \backslash A^{\prime}$ such that $\left.f\right|_{U \cap A}$ is an embedding. In this case, $\left.f\right|_{U \backslash A}$ is also an embedding, and $f(U \cap A) \cap f(U \backslash A)=\varnothing$. Thus, $\left.f\right|_{U}$ is an injection, $\left.f\right|_{X \backslash A^{\prime}}$ is a local injection, and by Lemma 5 it is an immersion.

Theorem 4. Suppose that

- $X$ is regular; $A \subset X$ is closed; for any point a of the boundary of $A$ and for its arbitrary neighborhood $U$, in $X$ there exists a neighborhood $U^{\prime} \subset U$ of the point a such that $U^{\prime} \backslash A$ is pathconnected;

- $B \subset Y$; for any point $b$ of the boundary of $B$ and for its arbitrary neighborhood $V$, in $Y$ there exists a neighborhood $V^{\prime} \subset V$ of the point $b$ such that $V^{\prime} \backslash B$ is connected and simply connected;

- $f \in C(X, A, X \backslash A ; Y, B, Y \backslash B)$ is a proper map; $\left.f\right|_{A \rightarrow B}$ and $\left.f\right|_{X \backslash A \rightarrow Y \backslash B}$ are local homeomorphisms.

Then $f$ is a covering.

Proof. By virtue of Lemma $1,\left.f\right|_{A \rightarrow B}$ is a covering over every connected component of $B$, and $\left.f\right|_{X \backslash A \rightarrow Y \backslash B}$ is a covering over every connected component of $Y \backslash B$.

Suppose that $a$ lies on the boundary of $A, b=f(a), V$ is neighborhood of $b$ in $Y$ such that $V \backslash B$ is connected and simply connected, $U$ is neighborhood of $a$ in $f^{-1}(V)$ such that $U \backslash A$ is pathconnected, and $\left.f\right|_{U \cap A}$ is an embedding. We see that $\left.f\right|_{U \backslash A}$ is also an embedding, and $f(U \cap A) \cap$ $f(U \backslash A)=\varnothing$. Thus, $\left.f\right|_{U}$ is an injection, $f$ is a local injection, and by Lemma 5 it is an immersion.

Let us show that $f$ is open. It is sufficient to prove that for any point $a$ on the boundary of $A$ and for an arbitrarily small neighborhood $U \ni a$ in $X$, the point $b=f(a)$ is contained in the interior of $f(U)$ in $Y$. Without loss of generality, we can choose $U$ so that $\left.f\right|_{\bar{U}}$ is an embedding and $U \backslash A$ is connected. Suppose that $b \notin \operatorname{int}_{Y} f(U)$. Then for any neighborhood $V \ni b$ we have $(V \backslash B) \backslash(f(U) \backslash B) \neq \varnothing$, because $f(U)$ contains a neighborhood of $b$ in $B$. If $V \backslash B$ is connected, then its intersection with the boundary of the set $f(U) \backslash B$ is nonempty, because $f(U) \backslash B=f(U \backslash A)$ is nonempty and open in $Y \backslash B$. Suppose that $\left\{y_{i}\right\}$ is a sequence of points of the boundary of $f(U)$ converging to $b$ and not lying in $B$. As $y_{i} \in \overline{f(U)} \backslash B$ and $\left.f\right|_{X \backslash A \rightarrow Y \backslash B}$ is a proper covering, $f^{-1}\left(y_{i}\right) \cap \bar{U}=\left\{x_{i}\right\}$ for some points $x_{i} \in \bar{U} \backslash(U \cup A)$. We have $\bigcup\left\{x_{i}\right\} \cup\{a\}=f^{-1}(K) \cap \bar{U}$, where $K=\bigcup\left\{y_{i}\right\} \cup\{b\}$. The set $K$ is compact because $f$ is proper. For any limit point $x^{\prime}$ of the sequence $\left\{x_{i}\right\}, f\left(x^{\prime}\right)=\lim y_{i}=b$, so that $\lim x_{i}=a$. However, $a$ cannot be a limit point of the boundary of the open set $U \ni a$. This contradiction shows that $b \in \operatorname{int} f(U)$.

Thus, $f$ is an open immersion, i.e., a local homeomorphism. Then by Lemma $1 f$ is a covering. 


\section{TOPOLOGICAL MANIFOLDS}

Definition 2. A Hausdorff topological space $M$ with a countable base is called an $n$ dimensional topological manifold if any point $x \in M$ has a neighborhood homeomorphic to space $\mathbb{R}^{n}$ or half-space $\mathbb{R}_{+}^{n}=\left\{\left(x_{1}, \ldots, x_{n}\right): x_{i} \in \mathbb{R}, x_{1} \geq 0\right\}$.

Points $x \in M$ having a neighborhood homeomorphic to $\mathbb{R}^{n}$ are called interior points. The subspace of $M$ consisting of points that are not interior is called the boundary $\partial M$ of the manifold $M$. If $\partial M=\varnothing$, then $M$ is called a manifold without boundary. The boundary of an $n$-dimensional manifold is an $(n-1)$-dimensional manifold without boundary.

If a subspace $A \subset M$ of the manifold $M$ is itself a manifold, then it is called a submanifold of $M$.

Let us first present two obvious corollaries of the results obtained in the previous section.

Theorem 5. Suppose that $M$ and $N$ are connected topological manifolds of equal dimensions, $f: M \rightarrow N$ is a continuous proper map, $A \subset M$ is closed, $\operatorname{int}_{M} A=\operatorname{int}_{N} f(A)=\varnothing,\left.f\right|_{M \backslash A}$ is a local homeomorphism, and $\left.f\right|_{M \backslash f^{-1}(f(A))}$ is an injection. In this situation,

(1) if $\left.f\right|_{A}$ is an immersion, then $f$ is also an immersion;

(2) if $\left.f\right|_{A}$ is an injection, then $f$ is an embedding;

(3) if $\left.f\right|_{A}$ is an injection and $N \backslash f(A)$ is connected, then $f$ is a homeomorphism.

Proof. This is a corollary of Theorem 3.

Theorem 6. Suppose that $M$ and $N$ are connected topological manifolds of equal dimensions, $f: M \rightarrow N$ is a continuous proper map, $A \subset M$ is closed, $\operatorname{int}_{M} A=\operatorname{int}_{N} f(A)=\varnothing, N \backslash f(A)$ is connected, $\left.f\right|_{M \backslash A}$ is a local homeomorphism, and one of the following two conditions is fulfilled:

- for some point $y_{0} \in N \backslash f(A)$ its preimage $f^{-1}\left(y_{0}\right)$ is a singleton, or

- $M \backslash A$ is connected, and $N \backslash f(A)$ is simply connected.

In this situation, if $\left.f\right|_{A}$ is an immersion, then $f$ is also an immersion; if $\left.f\right|_{A}$ is an injection, then $f$ is a homeomorphism.

Proof. This is a corollary of Lemmas 2 and 3 and Theorem 3.

Theorem 7. Suppose that $M$ and $N$ are topological manifolds of equal dimensions, $f \in$ $C(M, \partial M ; N, \partial N)$, and $\left.f\right|_{\partial M}$ and $\left.f\right|_{M \backslash \partial M}$ are immersions. Then $f$ is a local homeomorphism.

Proof. If $f$ is a proper map, then $f$ is a local homeomorphism by Theorem 4 (if we set $X=M$, $A=\partial M, Y=N$, and $B=\partial N)$. Let us prove the theorem in the general case.

The boundaries $\partial M$ and $\partial N$ are topological manifolds of equal dimensions without boundary, and by the domain invariance theorem [6] $\left.f\right|_{\partial M \rightarrow \partial N}$ is a local homeomorphism. By the same theorem, $f(M \backslash \partial M) \subset N \backslash \partial N$, and $\left.f\right|_{M \backslash \partial M \rightarrow N \backslash \partial N}$ is also a local homeomorphism.

Let us divide the proof into two stages: (1) $f$ is open and (2) $f$ is a local homeomorphism.

1. Let us prove that for any point $x_{0} \in \partial M$ and for its arbitrarily small neighborhood $U$ the point $y_{0}=f\left(x_{0}\right)$ has a neighborhood $V$ contained in $f(U)$.

Suppose that $x_{0} \in \partial M$ and $U$ is a neighborhood of $x_{0}$ in $M$ such that $\left.f\right|_{U \cap \partial M \rightarrow \partial N}$ is an embedding. Let us consider an $n$-dimensional simplex $\Delta^{n}, n=\operatorname{dim} M$, embedded in $U$ so that one of its $(n-1)$-dimensional faces $\Delta^{n-1}$ is embedded in $U \cap \partial M$ and contains $x_{0}$ in its relative interior, and the remaining part $\tilde{\Delta}^{n}=\Delta^{n} \backslash \Delta^{n-1}$ is embedded in $U \backslash \partial M$. Then $x_{0} \in \operatorname{int}_{M} \Delta^{n}$. 
Let $V$ be a connected neighborhood of the point $y_{0}=f\left(x_{0}\right)$ in $N$ not intersecting $f(Q), Q=$ $\partial \Delta^{n} \backslash\left(\Delta^{n-1} \backslash \partial \Delta^{n-1}\right)$, and such that $V \cap \partial N \subset f\left(\Delta^{n-1}\right)$ (such a neighborhood exists because $Q$ is compact). Suppose that $V \nsubseteq f\left(\Delta^{n}\right)$. Then $V \backslash \partial N \nsubseteq f\left(\Delta^{n}\right)$ too. As $f^{-1}(V) \cap \Delta^{n}$ is nonempty (contains $x_{0}$ ) and open in $\Delta^{n}$, we have $f^{-1}(V) \cap(M \backslash \partial M) \cap \Delta^{n} \neq \varnothing$ and $V \cap(N \backslash \partial N) \cap f\left(\Delta^{n}\right) \neq \varnothing$. Thus, in $V \backslash \partial N$ there are both points belonging to $f\left(\Delta^{n}\right)$ and points not belonging to it. Then the boundary $\Gamma$ of the set $f\left(\Delta^{n}\right)$ in $N$ must intersect $V \backslash \partial N$. Let $y \in(V \backslash \partial N) \cap \Gamma$. Since $f\left(\Delta^{n}\right)$ is compact, it follows that $\Gamma \subset f\left(\Delta^{n}\right)$ and $y=f(x)$ for some $x \in \tilde{\Delta}^{n}$. As $(V \backslash \partial N) \cap f\left(\partial \Delta^{n}\right)=\varnothing$, we have $x \in \Delta^{n} \backslash \partial \Delta^{n}$. However, $f\left(\Delta^{n} \backslash \partial \Delta^{n}\right)$ is open in $N \backslash \partial N$, because $\left.f\right|_{M \backslash \partial M \rightarrow N \backslash \partial N}$ is a local homeomorphism; therefore, $y=f(x)$ lies in the interior of $f\left(\Delta^{n}\right)$ in $N$, which contradicts the suggestion $x \in \Gamma$. Thus, $V \subset f\left(\Delta^{n}\right) \subset f(U)$, and $f$ is open.

2. Since $\left.f\right|_{M \backslash \partial M \rightarrow N \backslash \partial N}$ is a local homeomorphism and $M \backslash \partial M$ is open in $M$, it is sufficient to prove that for any $x_{0} \in \partial M$ there exists a neighborhood of $x_{0}$ in $M$ such that the restriction of $f$ to this neighborhood is an embedding.

Set $U^{\prime}=f^{-1}(V) \cap \Delta^{n}=f^{-1}(V) \cap\left(\Delta^{n} \backslash Q\right)$. As $f^{-1}(V)$ and $\Delta^{n} \backslash Q$ are open in $M$, the set $U^{\prime}$ is open in $M$. The map $\left.f\right|_{U^{\prime} \rightarrow V}$ is surjective and proper, because for any compact set $K \subset V$ its preimage $\left(\left.f\right|_{U^{\prime} \rightarrow V}\right)^{-1}(K)=f^{-1}(K) \cap U^{\prime}=f^{-1}(K) \cap \Delta^{n}$ is a closed subset of the compact set $\Delta^{n}$; hence, it is compact itself.

Consider an $n$-dimensional simplex $\sigma^{n}$ embedded in $V$ so that one of its $(n-1)$-dimensional faces $\sigma^{n-1}$ is embedded in $\partial N$ and contains $y_{0}$ in its relative interior, and the remaining part $\tilde{\sigma}^{n}=\sigma^{n} \backslash \sigma^{n-1}$ is embedded in $V \backslash \partial N$. Recall that $U$ has been chosen so that $\left.f\right|_{U \cap \partial M \rightarrow \partial N}$ is an embedding; thus, $\left.f\right|_{U^{\prime} \cap \partial M \rightarrow V \cap \partial N}$ is a homeomorphism. This means that $f$ defines a homeomorphism between $\sigma^{n-1}$ and some $(n-1)$-dimensional simplex $\rho^{n-1}$ embedded in $U^{\prime} \cap \partial M$.

As $\left.f\right|_{U^{\prime} \backslash \partial M \rightarrow V \backslash \partial N}$ is a covering (by Lemma 1) and $\tilde{\sigma}^{n}$ is simply connected, its preimage $\left(\left.f\right|_{U^{\prime} \backslash \partial M \rightarrow V \backslash \partial N}\right)^{-1}\left(\tilde{\sigma}^{n}\right)$ is homeomorphic to a disjoint union of one or several copies of $\tilde{\sigma}^{n}$ : $f^{-1}\left(\tilde{\sigma}^{n}\right) \cong J \times \tilde{\sigma}^{n}$, where $J$ is a set of indices with a discrete topology. Thus, we have obtained homeomorphisms $\tilde{g}_{j}: \tilde{\sigma}^{n} \rightarrow U^{\prime} \backslash \partial M, j \in J$, such that $f \circ \tilde{g}_{j}=\mathrm{id}$, and $\operatorname{Im} \tilde{g}_{i} \cap \operatorname{Im} \tilde{g}_{j}=\varnothing$ for $i \neq j$. The homeomorphisms $\tilde{g}_{j}$ can be continued to the homeomorphisms $g_{j}: \sigma^{n} \rightarrow U^{\prime}$ so that $f \circ g_{j}=$ id.

Thus, the closed neighborhood $f^{-1}\left(\sigma^{n}\right)$ of the point $x_{0}$ is homeomorphic to the quotient space of $J \times \sigma^{n}$ over the map $J \times \sigma^{n-1} \rightarrow \sigma^{n-1}$ (i.e., every copy of the $n$-simplex $j \times \sigma^{n}$ is attached to one copy of $\sigma^{n-1}$ over the standard embedding $\left.\sigma^{n-1} \rightarrow \sigma^{n}\right)$. This is compatible with the condition that a neighborhood of the point $x_{0}$ in $M$ is homeomorphic to half-space $\mathbb{R}_{+}^{n}$ if and only if the set of indices $J$ is a singleton.

Thus, a restriction of $f$ to some neighborhood of an arbitrary point $x_{0} \in \partial M$ is an embedding and $f$ is a local homeomorphism.

Theorem 8. Suppose that $M$ and $N$ are connected topological manifolds of equal dimensions, $f \in C(M, \partial M ; N, \partial N)$ is a proper map, and $\left.f\right|_{\partial M}$ and $\left.f\right|_{M \backslash \partial M}$ are immersions. Then $f$ is a covering.

If one of the following conditions is satisfied

- $N$ is simply connected, or

- there exists a point in $N$ with a one-point preimage, then $f$ is a homeomorphism.

Proof. This is a corollary of Theorem 7 and Lemmas 1-3 (and also a corollary of Theorem 4 and Lemmas 2-3). 
The following result is an evident corollary of Theorem 8; it is stated here as a separate statement due to the presence of its analogs for bounded domains in $\mathbb{R}^{n}$ in the papers $[3,4]$ and the absence of complete proofs of the corresponding theorems in those papers.

Theorem 9. Suppose that $M$ and $N$ are connected topological manifolds of equal dimensions with nonempty boundaries, $f \in C(M, \partial M ; N, \partial N)$ is a proper map, $\left.f\right|_{\partial M \rightarrow \partial N}$ is an injection, and $\left.f\right|_{M \backslash \partial M}$ is an immersion. Then $f$ is a homeomorphism.

Proof. As $f(M \backslash \partial M) \subset N \backslash \partial N$ and $\left.f\right|_{\partial M \rightarrow \partial N}$ is an injection, there exists $y_{0} \in \partial N$ with a singleton preimage. By Lemma $4,\left.f\right|_{\partial M}$ is an immersion. It now remains to apply Theorem 8.

In the previous theorems, it was required that the image of the boundary of $M$ be contained in the boundary of $N$; we now want to avoid this condition.

Theorem 10. Suppose that $M$ and $N$ are connected topological manifolds of equal dimensions, $N$ is simply connected, $\partial M$ is compact, $f: M \rightarrow N$ is a proper immersion, and the restriction of $f$ to every connected component of $\partial M$ is an injection. Then $f$ is an embedding.

This theorem is a generalization of Lemma 1 from [8] (which involves smooth compact manifolds $M$ and $N$ and a local diffeomorphism $f: M \rightarrow N$; in addition, $\partial N=\varnothing$ ). Let us use the idea of the proof from [8]: we cut $N$ along the images of the connected components of $\partial M$ and attach the emerging "caps" to $M$ along the corresponding components of $\partial M$. However, in our situation additional difficulties arise because we are within the framework of not smooth, but topological category (for example, we do not know whether the images of the connected components of $\partial M$ are locally flat submanifolds of $N$ ) and $N$ is not necessarily closed. In particular, we will need the following lemma.

Lemma 6. Suppose that $M$ and $N$ are topological manifolds, $\partial M$ is compact, $f: M \rightarrow N$ is an immersion, and the restriction of $f$ to $\partial M$ is an embedding. Then there exists a neighborhood $U \supset \partial M$ in $M$ such that the restriction of $f$ to $U$ is also an embedding.

Note that here, in contrast to other theorems of this section, the equality of dimensions of $M$ and $N$ is not needed.

Proof. As we are interested in the behavior of $f$ only in a neighborhood of $\partial M$, and $\partial M$ has a collar in $M$ [9], we can assume, without loss of generality, that $M=\partial M \times[0,1)$. Then the statement being proved is a corollary of the following fact.

Lemma 7. Suppose that $A$ and $N$ are metric spaces, $A$ is compact, $M=A \times[0,1), f: M \rightarrow N$ is an immersion, and $\left.f\right|_{A \times\{0\}}$ is an embedding. Then there exists a neighborhood $U \supset A \times\{0\}$ in $M$ such that the restriction of $f$ to $U$ is also an embedding.

Proof. Let us define a metric on $M$ by the equality $\rho\left((x, t),\left(x^{\prime}, t^{\prime}\right)\right)=\rho\left(x, x^{\prime}\right)+\left|t-t^{\prime}\right|$ (we will denote the metrics on $A, M$, and $N$ by the same letter $\rho$ for the sake of convenience; it will always be clear from the context which space is being discussed). Let us denote by $B_{a}(x)$ the open ball of radius $a$ centered at a point $x$.

Since $f$ is an immersion, for any point $x \in A$ there exists $a \in(0,1)$ such that $\left.f\right|_{B_{a} \times[0, a)}$ is an embedding. As $A$ is compact, it can be covered by a finite number of balls $B_{a_{i}}\left(x_{i}\right)$; we set $a=\min a_{i}$.

The set $\{(x, y): x, y \in A, \rho(x, y) \geq a\} \subset A^{2}$ is compact, and the continuous function $(x, y) \mapsto$ $\rho(f(x, 0), f(y, 0))$ attains here its minimum value $d>0$. Since $f$ is uniformly continuous on the compact set $A \times[0, a]$, it follows that

$$
\exists \epsilon \in(0, a) \forall z, z^{\prime} \in A \times[0, a] \quad \rho\left(z, z^{\prime}\right)<2 \epsilon \Rightarrow \rho\left(f(z), f\left(z^{\prime}\right)\right)<d / 2 .
$$


For any pair of points $(x, t),\left(x^{\prime}, t^{\prime}\right) \in A \times[0,2 \epsilon)$, two variants are possible:

- either $\rho\left(x, x^{\prime}\right)<a$, and then $f(x, t) \neq f\left(x^{\prime}, t^{\prime}\right)$ by the choice of $a$,

- or $\rho\left(x, x^{\prime}\right) \geq a$ and then $\rho\left(f(x, t), f\left(x^{\prime}, t^{\prime}\right)\right)>\rho\left(f(x, 0), f\left(x^{\prime}, 0\right)\right)-d / 2-d / 2 \geq 0$.

Thus, $\left.f\right|_{A \times[0,2 \epsilon)}$ is injective, $\left.f\right|_{A \times[0, \epsilon]}$ is an embedding, and $\left.f\right|_{A \times[0, \epsilon)}$ is also an embedding.

Proof of Theorem 10. The boundary $\partial M$ has a collar in $M$ [9]; i.e., there exists an embedding $\varphi: \partial M \times[0,1) \hookrightarrow M$ such that $\varphi(x, 0)=x$ for $x \in \partial M$. By Lemma $6, \varphi$ can be selected in such a way that the restriction of $f$ to every connected component of $\operatorname{Im} \varphi$ is an embedding. Let us define an isotopy $H:[0,1) \times M \rightarrow M$ by the formula

$$
H_{t}(x)= \begin{cases}x, & x \notin \operatorname{Im} \varphi \\ \varphi(q, s+t(1-s)), & x=\varphi(q, s) .\end{cases}
$$

In this case, $H_{0}=\mathrm{id}$, and $H_{t}$ is an embedding $M \hookrightarrow M$ for any $t \in[0,1)$.

Let us set $F_{t}=f \circ H_{t}$ and fix an arbitrary $t \in(0,1)$.

Let $A$ be a connected component of $\partial M$, and let $B=F_{t}(A)$. By construction, $\left.F_{t}\right|_{A \rightarrow B}$ is a homeomorphism, $B \subset N \backslash \partial N$. As $N$ is simply connected and $B$ is a connected closed submanifold of $N \backslash \partial N$ of codimension 1, we conclude that $N \backslash B$ falls into two connected components. For $U=\varphi(A \times[0,1))$, the image $F_{t}(U \backslash A)$ is connected and does not intersect $B$; hence, it is contained in one of the connected components of $N \backslash B$. Let us denote this component by $N_{1}$ and the second component by $N_{2}$. The set $N_{2} \cup B$ contains the collar $f \circ \varphi(A \times(0, t])$ of the submanifold $B=$ $f \circ \varphi(A \times\{t\})$, and thus $N_{2} \cup B$ is an $n$-dimensional topological manifold with boundary; one connected component of the boundary is $B$, and the remaining components are contained in $\partial N$. Let us attach the manifold $N_{2} \cup B$ to $M$ along $A$ by means of the homeomorphism $\left.F_{t}\right|_{A \rightarrow B}$, and continue $F_{t}$ to the obtained manifold, assuming that $\left.F_{t}\right|_{N_{2}}=$ id.

Having carried out this procedure successively for all connected components of $\partial M$ (there are a finite number of them because $\partial M$ is compact), we get the embedding $i_{t}: M \hookrightarrow \tilde{M}_{t}$ and immersion $\tilde{F}_{t}: \tilde{M}_{t} \rightarrow N$; in this case, the restriction of $\tilde{F}_{t}$ to every connected component of $\partial \tilde{M}_{t}$ is an embedding to $\partial N$. By construction, $\tilde{F}_{t}$ is a proper map. By Theorem $8, \tilde{F}_{t}$ is a homeomorphism and its restriction $\left.\tilde{F}_{t}\right|_{M}=F_{t}$ is an embedding.

Thus, for any $t \in(0,1)$ the map $F_{t}: M \rightarrow N$ is an embedding. As $\bigcup_{t>0} H_{t}(M)=M \backslash \partial M$, the restriction $\left.f\right|_{M \backslash \partial M}$ is also an embedding, and $f(M \backslash \partial M) \cap f(\partial M)=\varnothing$.

Assume that there exists a pair of different points $x_{0}, x_{1} \in \partial M$ with coinciding images $f\left(x_{0}\right)=$ $f\left(x_{1}\right)$. Suppose that $\gamma$ is a non-self-intersecting path in $M$ that connects $x_{0}$ with $x_{1}$ and lies in $M \backslash \partial M$ with the exception of its endpoints; $A$ is a connected component of $\partial M$ containing $x_{0}$. Then $\operatorname{Im}(f \circ \gamma)$ is a circle in $N$ and the index of its intersection with $f(A)$ is 1 , which contradicts the simple connectedness of $N$.

Thus, $\left.f\right|_{\partial M}$ is injective, $f$ is also injective, and by Lemma $4 f$ is an embedding.

\section{CELL DECOMPOSITIONS}

Definition 3. A decomposition of a topological space $X$ is called a cell decomposition if for any element of the decomposition (an open cell) $e$ : (1) a nonnegative integer $k$ (the dimension of $e$ ) is given, and (2) there exists a continuous map $\varphi$ of the closed disk $D^{k}$ to $X$ such that the restriction of $\varphi$ to the interior of $D^{k}$ is a homeomorphism on $e$, and the image of the restriction 
of $\varphi$ to the boundary of $D^{k}$ is contained in the union of cells with dimensions less than $k$ (such a map $\varphi$ is called a characteristic map for the cell $e$ ).

A Hausdorff topological space $X$ endowed with a cell decomposition is called a $C W$-complex if two conditions are satisfied: (C) the closure of every cell intersects only a finite number of cells, and (W) $A \subset X$ is closed if and only if the intersection of $A$ with the closure of every cell is closed.

The dimension of a CW-complex is the upper bound of dimensions of its cells. A CW-complex is called finite if it consists of a finite number of cells. The $k$-dimensional skeleton (the $k$-skeleton) of a CW-complex $X$ is the union $X_{k}$ of open cells of $X$ of dimensions at most $k$. A $C W$-subcomplex of a CW-complex is its subset that together with any of its points contains the closure of the cell containing this point.

\section{Codimension 1}

Theorem 11. Suppose that $M$ and $N$ are connected topological $n$-dimensional manifolds, and $A \subset M$ is closed in $M$ and has a structure of a $C W$-complex of dimension at most $n-1$. Then the following conditions are equivalent:

(1) $f: M \rightarrow N$ is a homeomorphism;

(2) $f \in C(M, \partial M ; N, \partial N)$ is a proper map, the restrictions of $f$ to $M \backslash A$ and to every open cell of $A$ are immersions, and $\left.f\right|_{M \backslash f^{-1}(f(A))}$ is an injection.

Proof. The implication $(1) \Rightarrow(2)$ is obvious. Let us prove the implication $(2) \Rightarrow(1)$.

As $M$ has a countable base, $A$ consists of at most a countable number of cells. In this case, $f(A)$ is a union of at most a countable number of submanifolds of $N$ of nonzero codimension. Every submanifold is nowhere dense in $N$; hence, $f(A)$ has an empty interior in $N$. Similarly, $A$ is a union of at most a countable number of disks of nonzero codimension embedded in $M$, and int $M=\varnothing$. By Theorem 2, $\left.f\right|_{M \backslash A}$ is an open embedding, so $f(A) \cap f(M \backslash A)=\varnothing$.

Let us denote by $A_{k}$ the $k$-skeleton of $A$.

Suppose that $\left.f\right|_{M \backslash A_{k}}$ is an open embedding. As $\left.f\right|_{A_{k} \backslash A_{k-1}}$ is an immersion, by assertion 3 of Theorem 3 the restriction $\left.f\right|_{M \backslash A_{k-1}}$ is an immersion. As $M \backslash A_{k-1}$ is an $n$-dimensional manifold with boundary $\partial\left(M \backslash A_{k-1}\right) \subset \partial M$, by Theorem 7 the restriction $\left.f\right|_{M \backslash A_{k-1}}$ is a local homeomorphism. By Theorem 2, $\left.f\right|_{M \backslash A_{k-1}}$ is an open embedding.

By induction, we find that $f$ is an open embedding. By Lemma $1, f$ is a homeomorphism.

Theorem 12. Suppose that $M$ and $N$ are connected topological $n$-dimensional oriented manifolds, $A \subset M$ is closed in $M$ and has a structure of a $C W$-complex of dimension at most $n-1$, and $A \cap \partial M$ is a $C W$-subcomplex of $A$. Then the following conditions are equivalent:

(1) $f: M \rightarrow N$ is an orientation-preserving homeomorphism;

(2) $f \in C(M, \partial M ; N, \partial N)$ is a proper map, $\left.f\right|_{M \backslash A}$ is an orientation-preserving immersion, the restriction of $f$ to every open cell of $A$ is an immersion, and for some point $x_{0} \in M \backslash A$ the condition $f^{-1}\left(f\left(x_{0}\right)\right)=\left\{x_{0}\right\}$ is satisfied.

Proof. The implication $(1) \Rightarrow(2)$ is evident; let us prove $(2) \Rightarrow(1)$.

Without loss of generality, we can assume that $\partial M=\partial N=\varnothing$, because the general case reduces to this one by passing to the doubles $\mathcal{D} f: \mathcal{D} M \rightarrow \mathcal{D} N$. Here $\mathcal{D}$ is the doubling functor, i.e., the gluing of two copies of a manifold along the boundary (in this case, one of the copies retains its orientation and the orientation of the other is changed to the opposite one). As $A \cap \partial M$ is a CWsubcomplex of $A$, we can define the double of $A$ as the closed $\mathrm{CW}$-complex $\mathcal{D} A \subset \mathcal{D} M$ : we leave 
the cells lying in $A \cap \partial M$ in one copy and double all other cells (characteristic maps for doubled cells are defined in an obvious way).

As $f$ is a proper map, the degree of $f$ is defined, and $f_{*}\left(o_{f^{-1}(y)}\right)=(\operatorname{deg} f) o_{y}$ for any $y \in N$, where $o_{y} \in H_{n}(N, N \backslash\{y\})$ and $o_{f^{-1}(y)} \in H_{n}\left(M, M \backslash f^{-1}(y)\right)$ are generators of corresponding relative homological groups [6]. By the hypothesis, $f^{-1}\left(f\left(x_{0}\right)\right)=\left\{x_{0}\right\}$, and $f$ is an orientation-preserving homeomorphism in a neighborhood of $x_{0}$; thus, $\operatorname{deg} f=1, f$ is surjective, and $\left.f\right|_{M \backslash f^{-1}(f(A)) \rightarrow N \backslash f(A)}$ is a homeomorphism.

It remains to apply Theorem 11.

\section{Codimension 2}

Theorem 13. Suppose that $M$ and $N$ are connected topological $n$-dimensional manifolds, $A \subset M$ has the structure of a finite $C W$-complex of dimension at most $n-2, f \in C(M, \partial M ; N, \partial N)$ is a proper map, and the restrictions of $f$ to $M \backslash A$ and to every open cell of $A$ are immersions. Suppose that one of the following conditions is satisfied:

(1) there exists a point $x_{0} \in M \backslash A$ such that $f^{-1}\left(f\left(x_{0}\right)\right)=\left\{x_{0}\right\}$, or

(2) $N \backslash f(A)$ is simply connected.

Then $f: M \rightarrow N$ is a homeomorphism.

To prove this theorem, we will need a number of auxiliary results.

Let us recall the definition of the dimension $\operatorname{dim} X$ of a topological space $X$ and some of its important properties.

- $\operatorname{dim} X$ is the smallest integer $n \geq 0$ satisfying the following condition: any finite open cover of the space $X$ has a finite open refining cover of multiplicity at most $n+1$ ([10], II.2).

- For an arbitrary subspace $X_{0}$ of an absolutely normal space $X, \operatorname{dim} X_{0} \leq \operatorname{dim} X$ ([10], IV.8, Theorem 18).

- If a normal space $X$ is the sum of a countable number of its closed subsets the dimension of every one of which is at most $n$, then the dimension of the space $X$ itself is at most $n$ ([10], IV.7, Theorem 16).

- If $M$ is an $n$-dimensional connected topological manifold without boundary and the dimension of $A \subset M$ is at most $n-2$, then $M \backslash A$ is connected ([10], VIII.2, Theorem 2).

Lemma 8. Suppose that $A$ is a finite $C W$-complex whose dimension is at most $n-2, N$ is an $n$-dimensional topological manifold, $f: A \rightarrow N$ is a continuous map, and the restriction of $f$ to every open cell of $A$ is an immersion. In this situation, if $N$ is path-connected, then $N \backslash f(A)$ is also path-connected.

Proof. Let us denote the combination of open $i$-dimensional cells of $A$ by $E_{i}$.

Every point $x \in E_{i}$ has a neighborhood $U_{x}$ in $E_{i}$ homeomorphic to the $i$-dimensional disk and such that $\left.f\right|_{U_{x}}$ is an embedding. The cover $\left\{U_{x}: x \in E_{i}\right\}$ contains a countable subcover $\left\{U_{i, j}\right\}$ of the space $E_{i}$. Then $f(A)$ is covered by countably many disks $f\left(U_{i, j}\right)$ of dimension at most $n-2$; consequently, both $f(A)$ and its subspace $f(A) \backslash \partial N$ must have dimensions at most $n-2$. Thus, $(N \backslash \partial N) \backslash(f(A) \backslash \partial N)=N \backslash(\partial N \cup f(A))$ is connected.

Since $A$ is compact, the set $f(A) \backslash \partial N$ is closed in $N \backslash \partial N$ and $N \backslash(\partial N \cup f(A))$ is a topological manifold without boundary. Hence, the connectedness of $N \backslash(\partial N \cup f(A))$ implies its pathconnectedness.

For any point $y_{0} \in \partial N \backslash f(A)$, there exist a neighborhood in $N$ not intersecting $f(A)$ and a path connecting $y_{0}$ with a point from $N \backslash(\partial N \cup f(A))$; thus, $N \backslash f(A)$ is also path-connected. 
Lemma 9. Suppose that $M$ and $N$ are connected topological $n$-dimensional manifolds, $A \subset M$ is a finite $C W$-complex of dimension at most $n-2, f: M \rightarrow N$ is a continuous proper map, and the restrictions of $f$ to $M \backslash A$ and to every open cell of $A$ are immersions. Then $M \backslash f^{-1}(f(A))$ is path-connected.

Proof. By the statements proved in the previous lemma, $\operatorname{dim} f(A) \leq n-2$.

Let $x \in f^{-1}(f(A)) \backslash A \subset M \backslash A$. By the hypothesis, there exists a neighborhood $U_{x} \subset M \backslash A$ of $x$ such that $\left.f\right|_{U_{x}}$ is an embedding. Then $\operatorname{dim}\left(f^{-1}(f(A)) \cap U_{x}\right) \leq n-2$. Since $f^{-1}(f(A)) \backslash A$ is covered by at most a countable number of such neighborhoods, we have $\operatorname{dim}\left(f^{-1}(f(A)) \backslash A\right) \leq n-2$, and $\operatorname{dim} f^{-1}(f(A)) \leq n-2$.

As $f$ is proper, $f^{-1}(f(A))$ is compact.

Therefore, similarly to the proof of the previous lemma, we get that $M \backslash f^{-1}(f(A))$ is pathconnected.

Proof of Theorem 13. By Lemmas 8 and $9, N \backslash f(A)$ and $M \backslash f^{-1}(f(A))$ are path-connected. By Theorem 2, $\left.f\right|_{M \backslash f^{-1}(f(A))}$ is an embedding. It remains to apply Theorem 11.

\section{Cell Decompositions of Manifolds}

Definition 4. A cell decomposition of a topological manifold $M$ will be called nice if $\partial M$ is a CW-subcomplex of $M$.

Theorem 14. Suppose that $M$ and $N$ are connected topological $n$-dimensional oriented manifolds and a nice cell decomposition of $M$ is given. Then the following conditions are equivalent:

(1) $f: M \rightarrow N$ is an orientation-preserving homeomorphism;

(2) $f \in C(M, \partial M ; N, \partial N)$ is a proper map; the restriction of $f$ to every open cell of $M$ is an immersion; moreover, if a cell is n-dimensional, then this immersion preserves its orientation; and for some point $x_{0} \in\left(M \backslash M_{n-1}\right) \cup\left(\partial M \backslash M_{n-2}\right)$, where $M_{i}$ is the $i$-skeleton of $M$, the identity $f^{-1}\left(f\left(x_{0}\right)\right)=\left\{x_{0}\right\}$ is true.

Proof. 1. Assume that $x_{0} \in \partial M \backslash M_{n-2}$. Let us show that this case reduces to the case $x_{0} \in M \backslash M_{n-1}$.

Let $e^{n-1}$ be an open $(n-1)$-dimensional cell of $\partial M$ containing $x_{0}$. By the definition of a CWcomplex, if the closure of some cell intersects $e^{n-1}$, then this cell is $n$-dimensional. In addition, $M$ is locally compact; therefore, the cell decomposition of $M$ is locally finite. Hence, there exists a neighborhood $U \ni x_{0}$ in $M$ such that $U \backslash \partial M \subset \bigcup e_{i}^{n}$, where $\left\{e_{i}^{n}\right\}$ are some $n$-dimensional cells. Without loss of generality, we can assume that $U$ is homeomorphic to $\mathbb{R}_{+}^{n}$ and $U \cap \partial M \subset e^{n-1}$. As $e_{i}^{n}$ are open and $U \backslash \partial M$ is connected, $U \backslash \partial M$ is completely contained in one of these $n$-dimensional cells; let us denote this cell by $e^{n}$.

$U$ is an $n$-dimensional topological manifold with boundary and $\left.f\right|_{U} \in C(U, \partial U ; N, \partial N)$. The restrictions $\left.f\right|_{\partial U}$ and $\left.f\right|_{U \backslash \partial U}$ are immersions since $\partial U$ and $U \backslash \partial U$ are contained in the open cells $e^{n-1}$ and $e^{n}$, respectively. By Theorem $7,\left.f\right|_{U}$ is a local homeomorphism.

Let $U^{\prime} \in U$ be a neighborhood of $x_{0}$ such that $\left.f\right|_{U^{\prime}}$ is an embedding. Suppose that there exists a sequence $\left\{x_{i}\right\} \subset M \backslash U^{\prime}$ such that $f\left(x_{i}\right) \rightarrow y_{0}=f\left(x_{0}\right)$. As $\bigcup\left\{f\left(x_{i}\right)\right\} \cup\left\{y_{0}\right\}$ is compact and $f$ is proper, the sequence $\left\{x_{i}\right\}$ has a limit point $x \in M$. By the continuity of $f, f(x)=y_{0}$; since $f^{-1}\left(y_{0}\right)$ is a singleton, $x=x_{0}$. However, $x_{0}$ cannot be a limit point of the sequence of which all elements lie out of $U^{\prime}$. 
The obtained contradiction shows that there exists a neighborhood $V \ni y_{0}$ in $N$ such that $f^{-1}(V) \subset U$. However, in this case $\left.f\right|_{f^{-1}(V)}$ is an embedding, and, for any point $x_{0}^{\prime} \in f^{-1}(V) \cap$ $\left(M \backslash M_{n-1}\right)$, we have $f^{-1}\left(f\left(x_{0}^{\prime}\right)\right)=\left\{x_{0}^{\prime}\right\}$. Hence, the point $x_{0}$ in the hypothesis can be replaced by the point $x_{0}^{\prime} \in M \backslash M_{n-1}$.

2. Now let $x_{0} \in M \backslash M_{n-1}$. Set $A=M_{n-1}$ and apply Theorem 12 .

In the case when $M$ or $N$ is unoriented, we must additionally consider the behavior of $f$ in a neighborhood of $(n-1)$-dimensional cells.

Theorem 15. Suppose that $M$ and $N$ are connected topological $n$-dimensional manifolds, $M$ is compact, and a nice cell decomposition of $M$ is given. Then the following conditions are equivalent:

(1) $f: M \rightarrow N$ is a homeomorphism;

(2) $f \in C(M, \partial M ; N, \partial N)$ is a proper map, the restriction of $f$ to $M \backslash M_{n-2}$ and to every open cell of $M_{n-2}$ is an immersion, and the relation $f^{-1}\left(f\left(x_{0}\right)\right)=\left\{x_{0}\right\}$ holds for some point $x_{0} \in M \backslash M_{n-2}$.

Proof. Set $A=M_{n-2}$ and apply Theorem 13.

\section{TRIANGULATIONS}

Definition 5. A triangulation of a set is its cover by simplices such that (1) faces of any simplex of the cover are also simplices of the cover; (2) if one simplex of the cover is covered by another, then the first simplex is a face of the second simplex; and (3) the intersection of two overlapping simplices of the cover is also a simplex of the cover.

A simplicial space is a set endowed with a triangulation and with the topology induced by this triangulation (a subset is closed if and only if its intersection with any simplex of the triangulation is closed).

The following theorem is a corollary of Theorem 14 .

Theorem 16. Suppose that $M$ and $N$ are connected topological $n$-dimensional oriented manifolds, $K$ is a triangulation of $M$ (not necessarily a combinatorial one), $f \in C(M, \partial M ; N, \partial N)$ is a proper map, the restriction of $f$ to the relative interior of every simplex of the triangulation is an immersion, and the restriction of $f$ to the relative interior of every $n$-dimensional simplex preserves orientation. Suppose that for some point $x_{0} \in M$ lying in the union of the relative interiors of $n$-dimensional simplices of $M$ and $(n-1)$-dimensional simplices of $\partial M$ the relation $f^{-1}\left(f\left(x_{0}\right)\right)=\left\{x_{0}\right\}$ holds. Then $f: M \rightarrow N$ is a homeomorphism map $K$ to the triangulation of $N$.

Definition 6. A simplicial space $K$ is called an $n$-dimensional pseudomanifold if the following conditions are satisfied: (1) every simplex of $K$ is a face of some $n$-dimensional simplex of $K$, (2) every $(n-1)$-dimensional simplex of $K$ is a face of at most two $n$-dimensional simplices of $K$, and (3) any two $n$-dimensional simplices of $K$ can be included into a finite sequence of $n$-dimensional simplices of $K$ such that every two neighboring simplices of this sequence have a common $(n-1)$ dimensional face. The boundary $\partial K$ of an $n$-dimensional pseudomanifold $K$ is a subspace of $K$ formed by $(n-1)$-dimensional simplices of $K$ every one of which is a face of only one $n$-dimensional simplex of $K[11]$. 
Theorem 17. Let $K$ be a compact connected $n$-dimensional oriented pseudomanifold and let $N$ be a connected $n$-dimensional oriented topological manifold. Then the following conditions are equivalent:

(1) $K$ is a triangulated topological manifold and $f: K \rightarrow N$ is an orientation-preserving homeomorphism;

(2) $f \in C(K, \partial K ; N, \partial N)$; the restriction of $f$ to the relative interior of every simplex of $K$ is an immersion and for every $n$-dimensional simplex of $K$ this immersion preserves orientation; and the relation $f^{-1}\left(f\left(x_{0}\right)\right)=\left\{x_{0}\right\}$ holds for some point $x_{0} \in\left(K \backslash K_{n-1}\right) \cup\left(\partial K \backslash K_{n-2}\right)$, where $K_{i}$ is the $i$-skeleton of $K$.

To prove this theorem, we will need the following generalization of the domain invariance theorem to pseudomanifolds.

Lemma 10. Suppose that $K$ is an $n$-dimensional pseudomanifold, $U \subset K \backslash \partial K$ is open, and $f: U \rightarrow \mathbb{R}^{n}$ is an immersion. Then $f$ is a local homeomorphism and $U$ is a topological manifold.

Proof. It is sufficient to prove that if $f: U \rightarrow \mathbb{R}^{n}$ is an embedding for an open $U \subset K \backslash \partial K$, then $f(U)$ is open. The proof is similar to the proof of the domain invariance theorem in [6]. Let $x \in U$ and let $K^{\prime}$ be a subdivision of the triangulation $K$ such that the star $S$ of the point $x$ in the triangulation $K^{\prime}$ is contained in $U$. Denote the link of $x$ in $K^{\prime}$ by $L$; it is the boundary of $S$.

By the Aleksander-Pontryagin duality, the number of path-connected components of $\mathbb{R}^{n} \backslash f(L)$ is greater by one than the rank of the free $\mathbb{Z}_{2}$-module $\check{H}^{n-1}\left(L ; \mathbb{Z}_{2}\right)$ of Cech cohomologies of $L$ with coefficients in $\mathbb{Z}_{2}[6]$. Since $L$ is a connected closed $(n-1)$-dimensional pseudomanifold, $\check{H}^{n-1}\left(L ; \mathbb{Z}_{2}\right)=H^{n-1}\left(L ; \mathbb{Z}_{2}\right)=\mathbb{Z}_{2}$, and $\mathbb{R}^{n} \backslash f(L)=\left(\mathbb{R}^{n} \backslash f(S)\right) \cup f(S \backslash L)$ has two connected components.

Since $S$ is contractible, $\mathbb{R}^{n} \backslash f(S)$ is connected.

As $f(S \backslash L)$ is connected, it must be a connected component of $\mathbb{R}^{n} \backslash f(L)$. As $\mathbb{R}^{n} \backslash f(L)$ is open in $\mathbb{R}^{n}$, we conclude that $f(S \backslash L)$ is also open in $\mathbb{R}^{n}$. Thus, for any point $x \in U$ there exists its neighborhood $S \backslash L$ in $U$ such that $f(S \backslash L)$ is open in $\mathbb{R}^{n}$.

Proof of Theorem 17. The implication $(1) \Rightarrow(2)$ is obvious; let us prove $(2) \Rightarrow(1)$.

1. Let $K_{i}$ denote the $i$-skeleton of $K, K_{i-1}=\varnothing$. The case $x_{0} \in \partial K \backslash K_{n-2}$ reduces to the case $x_{0} \in K \backslash K_{n-1}$, as in the proof of Theorem 14. As in the proof of Theorem 12, we reduce the problem to the case $\partial K=\partial N=\varnothing$, passing to the doubles if necessary.

Set $N^{\prime}=N \backslash f\left(K_{n-2}\right)$ and $K^{\prime}=K \backslash f^{-1}\left(f\left(K_{n-2}\right)\right) \subset K \backslash K_{n-2}$. As $K_{n-2}$ is compact and $K \backslash K_{n-2}$ is a topological manifold, $K^{\prime}$ and $N^{\prime}$ are also $n$-dimensional topological manifolds without boundary. Let us consider the proper map $f^{\prime}=\left.f\right|_{K^{\prime} \rightarrow N^{\prime}}$. By Lemma $8, N^{\prime}$ is connected; thus, the degree of $f^{\prime}$ is defined. By the hypothesis, $\operatorname{deg} f^{\prime}=1$; hence, $\left.f\right|_{K \backslash f^{-1}\left(f\left(K_{n-1}\right)\right)}$ is an embedding. Applying assertion 2 of Theorem 2 to $X=K, A=K_{n-1}$, and $Y=N$, we find that $\left.f\right|_{K \backslash K_{n-1}}$ is an open map.

2. Assume that we have already proved that $\left.f\right|_{K \backslash K_{i}}$ is an open embedding for some $i \leq n-1$. Applying Theorem 3 to $X=K, A=K_{i}, A^{\prime}=K_{i-1}$, and $Y=N$, we find that $\left.f\right|_{K \backslash K_{i-1}}$ is an immersion. By Lemma 10, this immersion is a local homeomorphism. By Lemma $8, N \backslash f\left(K_{i-1}\right)$ is connected, and by the hypothesis of the theorem there exists a point in $N \backslash f\left(K_{i-1}\right)$ with a onepoint preimage. Thus, Theorem 2 is applicable to $X=K, A=K_{i}$, and $Y=N$, and we get that $\left.f\right|_{K \backslash K_{i-1}}$ is an open embedding.

Thus, by induction, $f=\left.f\right|_{K \backslash K_{-1}}$ is an open embedding. As $K$ is compact, $f$ is a homeomorphism. Since $K$ is homeomorphic to the topological manifold $N$, it is a topological manifold 
itself.

In the case when $N$ or $K$ is unoriented, the following formulation can be used.

Theorem 18. Suppose that $K$ is a compact connected $n$-dimensional pseudomanifold and $N$ is a connected $n$-dimensional topological manifold. Then the following conditions are equivalent:

(1) $K$ is a triangulated topological manifold and $f: K \rightarrow N$ is a homeomorphism;

(2) $f \in C(K, \partial K ; N, \partial N)$, the restriction of $f$ to $K \backslash K_{n-2}$ and to the relative interior of every simplex of $K_{n-2}$ is an immersion, and the relation $f^{-1}\left(f\left(x_{0}\right)\right)=\left\{x_{0}\right\}$ holds for some point $x_{0} \in$ $K \backslash K_{n-2}$.

Proof. As in the proof of the previous theorem, we reduce the general case to the case $\partial K=$ $\partial N=\varnothing$ and consider the proper map $f^{\prime}=\left.f\right|_{K^{\prime} \rightarrow N^{\prime}}$. The difference from the hypothesis of the previous theorem is that here the map $f^{\prime}$ is itself a local homeomorphism. By Lemmas 8 and 9 , the manifolds $K^{\prime}$ and $N^{\prime}$ are connected. By Theorem $8, f^{\prime}$ is a homeomorphism. By assertion 2 of Theorem $2,\left.f\right|_{K \backslash K_{n-2}}$ is an open embedding. It remains to repeat the argument from the second part of the proof of the previous theorem, with the difference that the induction is started with $i=n-2$ and not with $i=n-1$, as there.

\section{SMOOTH MANIFOLDS}

Definition 7. Let $M$ be an $n$-dimensional topological manifold. A chart of $M$ is a homeomorphism $\varphi$ of an open domain $U \subset M$ to $\mathbb{R}^{n}$ or to $\mathbb{R}_{+}^{n}$. Charts $\varphi: U \rightarrow V$ and $\varphi^{\prime}: U^{\prime} \rightarrow V^{\prime}$ are called $C^{r}$-compatible if the map $\varphi^{\prime} \circ \varphi^{-1}$ and the inverse map are $C^{r}$-smooth in their domains. A $C^{r}$-structure on $M$ is a cover of $M$ by pairwise $C^{r}$-compatible charts. Two $C^{r}$-structures on $M$ are considered equivalent if their union is also a $C^{r}$-structure.

A $C^{r}$-manifold (a manifold of smoothness class $C^{r}$ ) is an $n$-dimensional topological manifold $M$ with a $C^{r}$-structure defined on it.

A map $f: M \rightarrow M^{\prime}$, where $M$ and $M^{\prime}$ are $C^{r}$-manifolds, is called a smooth map of class $C^{r}$, or $C^{r}$-map, if for any pair of charts $\varphi: U \rightarrow V$ and $\varphi^{\prime}: U^{\prime} \rightarrow V^{\prime}\left(U \subset M, U^{\prime} \subset M^{\prime}\right)$ the map $\varphi^{\prime} \circ f \circ \varphi^{-1}$ is $C^{r}$-smooth in its domain. We will denote the set of $C^{r}$-maps $f: M \rightarrow M^{\prime}$ by $C^{r}\left(M, M^{\prime}\right)$.

A $C^{r}$-map $f: M \rightarrow N$ is called a $C^{r}$-immersion if its differential $\mathrm{d} f$ is nondegenerate $(\operatorname{rang}(\mathrm{d} f)=\operatorname{dim} M)$ at every point. An injective $C^{r}$-immersion is called a $C^{r}$-embedding. A bijective $C^{r}$-immersion is called a $C^{r}$-diffeomorphism.

Note that any $C^{r}$-immersion is (if we forget about the smooth structure) an immersion in terms of Definition 1, and every $C^{r}$-embedding is an embedding in terms of Definition 1 . That is why the results of Sections 2-4 can easily be reformulated for the smooth case (note that the direct proof of smooth and piecewise smooth versions is much easier than proofs of their topological analogs). Let us present the smooth versions of some of them (everywhere below, $r \geq 1$ ).

Theorem 19. Suppose that $M$ and $N$ are connected $C^{r}$-manifolds of equal dimensions, $f \in$ $C^{r}(M, \partial M ; N, \partial N)$ is a proper map, $\mathrm{d} f$ is nondegenerate on $M$, and one of two conditions is satisfied:

- $N$ is simply connected or

- there exists a point in $N$ with a one-point preimage.

Then $f$ is a $C^{r}$-diffeomorphism.

PROCEEDINGS OF THE STEKLOV INSTITUTE OF MATHEMATICS Suppl. 12008 
Theorem 20. Suppose that $M$ and $N$ are connected $C^{r}$-manifolds of equal dimensions with nonempty boundaries, $f \in C(M, \partial M ; N, \partial N) \cap C^{r}(M \backslash \partial M, N)$ is a proper map, $\mathrm{d} f$ is nondegenerate on $M \backslash \partial M$, and $\left.f\right|_{\partial M}$ is an injection. Then $f$ is a homeomorphism and the restriction $\left.f\right|_{M \backslash \partial M \rightarrow N \backslash \partial N}$ is a $C^{r}$-diffeomorphism. If $\mathrm{d} f$ is nondegenerate on $M$, then $f$ is a $C^{r}$ diffeomorphism.

Theorem 21. Suppose that $M$ and $N$ are connected $C^{r}$-manifolds of equal dimensions, $N$ is simply connected, $\partial M$ is compact, $f \in C^{r}(M, N)$ is a proper immersion, and the restriction of $f$ to every connected component of $\partial M$ is an injection. Then $\left.f\right|_{M \rightarrow f(M)}$ is a $C^{r}$-diffeomorphism.

Let $K$ be a simplicial space, and let $N$ be a $C^{r}$-manifold. The notions of a (piecewise smooth) $C^{r}$-map, $C^{r}$-immersion, and $C^{r}$-embedding are introduced in [12] for maps from $K$ to $N$, as well as the notion of a $C^{r}$-triangulation of the manifold $N$. The following result is proved there (Theorem 3.8):

Every nondegenerate $C^{r}$-map $f: K \rightarrow N$ that is a homeomorphism of the polyhedron $K$ to the manifold $N$ is a $C^{r}$-triangulation of this manifold.

The following results (oriented and unoriented versions) are obvious corollaries of this theorem and of our Theorems 17 and 18.

Theorem 22. Suppose that $K$ is a compact connected $n$-dimensional oriented pseudomanifold, $N$ is a connected $n$-dimensional oriented $C^{r}$-manifold, $f \in C(K, \partial K ; N, \partial N)$ is a nondegenerate $C^{r}$-map, and the restriction of $f$ to the relative interior of every $n$-dimensional simplex preserves orientation. Suppose that the relation $f^{-1}\left(f\left(x_{0}\right)\right)=\left\{x_{0}\right\}$ holds for some point $x_{0} \in$ $\left(K \backslash K_{n-1}\right) \cup\left(\partial K \backslash K_{n-2}\right)$. Then $f$ defines a $C^{r}$-triangulation of $N$.

Theorem 23. Suppose that $K$ is a compact connected $n$-dimensional pseudomanifold, $N$ is a connected n-dimensional $C^{r}$-manifold, $f \in C(K, \partial K ; N, \partial N)$ is a nondegenerate $C^{r}$-map, and the restriction of $f$ to $K \backslash K_{n-2}$ is a $C^{r}$-immersion. Suppose that the relation $f^{-1}\left(f\left(x_{0}\right)\right)=\left\{x_{0}\right\}$ holds for some point $x_{0} \in K \backslash K_{n-2}$. Then $f$ defines a $C^{r}$-triangulation of $N$.

\section{CONCLUSION}

The criteria proved in this paper may be used for finding characteristics of a continuous map given either globally on some manifold or independently on every cell or simplex into which this manifold is decomposed.

Some results of this work are generalizations of criteria formulated in the papers $[3,4]$ and widely used nowadays for designing grid generation algorithms.

Thus, Theorem 3 in [4] is a special case of Theorem 9 in this paper (and an even more special case of Theorem 8), and in our terms it reads as follows. Suppose that $\bar{\Omega} \subset \mathbb{R}^{n}$ is an n-dimensional $C^{1}$-manifold, $f \in C^{1}(\bar{\Omega}, \partial \bar{\Omega} ; \bar{\Omega}, \partial \bar{\Omega}),\left.f\right|_{\partial \bar{\Omega} \rightarrow \partial \bar{\Omega}}$ is a diffeomorphism, and $\left.f\right|_{\Omega}$ is a $C^{1}$-embedding; then $f$ is a homeomorphism. Note that in contrast to [4] in our results

- the smoothness of $f$ is not required;

- $f$ is not required to define a diffeomorphism of the boundary to the boundary (we only require it to map the boundary to the boundary injectively, or, in Theorem 8 , to be an immersion of the boundary in the boundary);

- $f$ is defined on an arbitrary topological manifold and it acts to an arbitrary topological manifold (but not to the initial domain or a domain homeomorphic to it).

Theorems 7-9 of paper [4] are corollaries of our Theorem 14. 
For example, in Theorem 7 of paper [4] the following is stated. Suppose that $\Omega$ is a bounded connected domain in $\mathbb{R}^{n}$ and a decomposition of $\bar{\Omega}$ to cells $\overline{\Omega_{i}}$ is given, with every cell being a convex domain in $\mathbb{R}^{n}, f \in C(\bar{\Omega}, \partial \bar{\Omega} ; \bar{\Omega}, \partial \bar{\Omega}),\left.f\right|_{\partial \bar{\Omega} \rightarrow \partial \bar{\Omega}}$ is a homeomorphism, and the restriction $f_{i}=\left.f\right|_{\overline{\Omega_{i}}}$ to the closure of every cell is a $C^{1}$-immersion. Suppose that for any point $x \in \bar{\Omega}$ the convex hull of the set of matrices $\left\{\left.\mathrm{d} f_{i}\right|_{x}: x \in \overline{\Omega_{i}}\right\}$ contains matrices with positive determinant only. Then $f$ is a homeomorphism. Note that the condition of the convexity of cells means that all interior faces - cells of nonmaximal dimensionality - are planar.

In Theorem 9 of paper [4], the same decomposition of $\bar{\Omega}$ to cells $\overline{\Omega_{i}}$ is considered, and it is proved there that $f \in C(\bar{\Omega}, \partial \bar{\Omega} ; \bar{\Omega}, \partial \bar{\Omega})$ is a homeomorphism if $\left.f\right|_{\partial \bar{\Omega} \rightarrow \partial \bar{\Omega}}$ is a homeomorphism and if for every closed cell $f_{i}=\left.f\right|_{\overline{\Omega_{i}}}$ is an orientation-preserving $C^{1}$-immersion such that its restriction to the boundary of this cell $\left.f\right|_{\partial \overline{\Omega_{i}}}$ is an embedding.

In contrast to these conditions, in Theorem 14 of this paper

- cells can have arbitrary form (there are no requirements of convexity, planar faces, etc.);

- the smoothness of the map $f$ is not required (the continuity is sufficient);

- a condition (the "nondegeneracy") is imposed on the behavior of $f$ only inside cells and faces but not on their closures, and not on the whole set of matrices from the convex hull, as described above;

- the restrictions of $f$ to the boundaries of cells are not required to be injective;

- the existence of only one point with a one-element preimage is sufficient (we do not require $f$ to map homeomorphically the boundary of the domain to the boundary);

- the map is defined on an arbitrary topological manifold (not on a closed domain in $\mathbb{R}^{n}$ ) and acts to an arbitrary topological manifold (not to the initial domain or a domain homeomorphic to it).

A number of theorems in $[3,4]$ are concerned with maps of the closure of an arbitrary bounded connected domain $\Omega \subset \mathbb{R}^{n}$ to itself. Unfortunately, two of these theorems are incorrect and the proofs of others are either incomplete or absent.

The fallacy of Theorem 4 in [3] is shown by the following counterexample: $\Omega=\{(\rho, \varphi) \in$ $\left.\mathbb{R}^{2}: 1 \leq \rho \leq 2, \varphi \neq 0\right\}$ is a bounded connected simply connected domain in $\mathbb{R}^{2}$ (here $(\rho, \varphi)$ are polar coordinates), and a smooth map $h: \bar{\Omega} \rightarrow \bar{\Omega}$ is given by the formula $(\rho, \varphi) \longmapsto(\rho, 2 \varphi)$. The domain $\Omega$ and map $h$ satisfy the hypothesis of this theorem, but $h$ is not a homeomorphism of $\bar{\Omega}$ to $\bar{\Omega}$. A counterexample to Theorem 6 in [3] is constructed similarly.

In the proofs of Theorem 3 in [3] and Theorems 3 and 5 in [4], the authors use an unjustified assumption that the intersection of the image of the domain and the image of its boundary is empty.

For example, in Theorem 3 in [3] the following is stated. Suppose that $\Omega \subset \mathbb{R}^{n}$ is a bounded connected domain, $h \in C^{1}(\bar{\Omega}, \partial \Omega ; \bar{\Omega}, \partial \Omega)$, $\operatorname{det} h^{\prime}>0$ on $\bar{\Omega}$, and $\left.h\right|_{\partial \Omega \rightarrow \partial \Omega}$ is a homeomorphism; then $h$ is a homeomorphism (here $\partial \Omega$ is the boundary of the domain $\Omega$ ). The proof of the surjectivity of $h$ is based on the assumption (which is not formulated explicitly) that for any point $x_{1} \in \Omega$ the vector field $\Phi_{1}(x)=h(x)-h\left(x_{1}\right)$ does not vanish on $\partial \Omega$. This assumption is fulfilled only when $h(\partial \Omega) \cap h(\Omega)=\varnothing$. However, it is neither proved nor discussed in [3]. The following example shows that this assumption is not fulfilled for an arbitrary local homeomorphism $h: \bar{\Omega} \rightarrow \bar{\Omega}$ that maps injectively the boundary of $\Omega$ to itself (i.e., when the bijectivity condition $\left.h\right|_{\partial \Omega \rightarrow \partial \Omega}$ is weakened to injectivity): $\Omega=(0,1) \times(0,1) \backslash\left\{2^{-2^{i}}: i \in \mathbb{N}\right\} \times\{1 / 2\} \subset \mathbb{R}^{2}, h(x, y)=\left(x^{2}, y\right)$.

Note that this gap in the proofs is connected with the fact that the boundary of $\Omega$ can have "interior" points, which are points lying in $\operatorname{int}_{\mathbb{R}^{n}} \bar{\Omega}$. If we forbid this possibility (i.e., if we consider only those domains $\Omega$ that coincide with the interior of their closure), then Theorem 3 in [3] and 
Theorems 1, 4, 5, and 6 in [4] are evident corollaries of Lemma 2 from [13] (one has to replace $X=\bar{\Omega}, Z=\varnothing, M_{n}=\mathfrak{M}_{n}=\mathbb{R}^{n}$ in the statement of this lemma).

\section{ACKNOWLEDGMENTS}

The paper was completed during my stay in the Institute des Hautes Études Scientifiques (France); I am grateful to this institute for the hospitality and wonderful work environment. I wish to express my thanks to P.E. Pushkar', E.G. Pytkeev, and the author of the blog [14] for useful advice, and to O.V. Ushakova for drawing my attention to the problem.

\section{REFERENCES}

1. O. V. Ushakova, Proc. Steklov Inst. Math. Suppl. 1, S78 (2004).

2. O. V. Ushakova, in Advances in Grid Generation, Ed. by O. V. Ushakova (Nova Science, New York, 2007), pp. $241-278$.

3. N. A. Bobylev, S. A. Ivanenko, and I. G. Ismailov, Mat. Zametki 60 (4), 593 (1996).

4. N. A. Bobylev, S. A. Ivanenko, and A. V. Kazunin, Zh. Vychisl. Mat. Mat. Fiz. 43 (6), 808 (2003).

5. M. F. Prokhorova, in Problems of Theoretical and Applied Mathematics (UrO RAN, Yekaterinburg, 2007), pp. 6569 [in Russian].

6. A. Dold, Lectures on Algebraic Topology (Springer-Verlag, Berlin, 1972; Mir, Moscow, 1976).

7. V. A. Rokhlin and D. B. Fuks, Beginner's Course in Topology: Geometric Chapters (Nauka, Moscow, 1977; Springer-Verlag, Berlin, 1984).

8. D. Novikov and A. Khovanskii, Mosc. Math. J. 6 (1), 135 (2006).

9. M. Brown, Ann. of Math. 75 (2), 331 (1962).

10. P. S. Aleksandrov and B. A. Pasynkov, Introduction to Dimension Theory. Introduction to the Theory of Topological Spaces and to General Dimension Theory (Nauka, Moscow, 1973) [in Russian].

11. E. Spanier, Algebraic topology (McGraw-Hill, New York, 1966; Mir, Moscow, 1971).

12. J. Mankres, Elementary Differential Topology (Princeton Univ., Princeton, NJ, 1963).

13. G. H. Meisters and C. Olech, Duke Math. J. 30 (1), 63 (1963).

14. http://sowa.livejournal.com 